\title{
Image Information Processing System
}

\author{
Young-Ku Kang ${ }^{\dagger \dagger}$ \\ Dept. of Computer Engineering, \\ Seoul National University of Technology
}

\begin{abstract}
Summary
This paper proposed that an advanced algorithm for selecting a seam line automatically which used to be selected by human operator for mosaicking images. The presented algorithm is based on the identification induced by the correlation and the direction of tie points. Tie points were selected from two geometrically distored spatial ortho-images. And the identification of selected tie points were represented by FOM. Additionally, four factors were taken into account for weights of the directed graph. These five factors were adjusted finely to work equally on selecting seam line. Then, the Dijkstra algorithm was executed repeatedly after each line adjustment. Using the seam line selected by this method, two spatial orthoimages were merged, and the effect of the identification of tie points was analyzed.
\end{abstract}

Key words: .

\section{Introduction}

The mosaic is a set of process that combines each image with an image remaining the continuance of feature. When an each spatial ortho-image is transformed into broad spatial ortho-image, mosaic must be achieved [1,2].

The spatial ortho-image is a type of perpendicular photographing that corrects the distortion by tilt observation and relief from central projection spatial image. Accordingly, the correlation location of a feature that appears on the spatial ortho-image is identical to the feature image and we can retrieve information such as distance, angle, coordinate and size. So, this may be applied to GIS and remote sensing [3, 4, 5].

The distortion correction of central projection is called as digital differential rectification and uses the surface height information of feature that is easy to discriminate between optical information such as camera location and pose at photographing and spatial image. However, the accuracy of optical and surface height information is limited by the orientation error of camera sensor, relief, curved surface, distortion by film and scanner and measurement error [6]. The accuracy limitation generates the distortion of spatial ortho-image that makes a difference between the object coordinate of map and pixel coordinate of spatial orthoimage, and unique feature that appears on two spatial ortho-images appears on different plane coordinate. Accordingly, method to simply merges two spatial ortho- images and make it mosaic incurs a feature discontinuity $[5,7,8]$.

A seam line distinguishes which pixel is used to mosaic result image in the overlapping area of two spatial orthoimages and is represented by a segment of a line that couples several peaks (called as junction), which are identical feature in two spatial ortho-image. In the process of mosaic, seam line must be defined. To combine one spatial ortho-image is a mosaic after cutting and revising two spatial ortho-images according to this seam line $[5,6$, 7].

In mosaic process, general seam line processing is to identify the identical feature and make the seam line in the overlapping area of both images by human. This borer line generation method has following problems, so automatic processing researches using computer algorithm are ongoing $[4,8,9]$.

Firstly, seam line processing costs a great deal on the time and working because human workers need to interfere.

Secondly, the errors that human incurs extend to the error of seam line.

Thirdly, it cannot make consistent result because each human worker generates the different results of seam line. This paper is devoted to propose practical seam line generation method using an algorithm to make a seam line automatically without human working.

\section{Mosaic automation theory}

Yehuda Afek tries to solve the problems in the process of mosaic by 6 steps in "Masaicking of Orthocertified aerial Images" [3] like figure 1.

\subsection{Global layout phase}

Global layout phase is to distinguish the overlapping area between two spatial ortho-images and set the coarse seam line like figure 2 . 


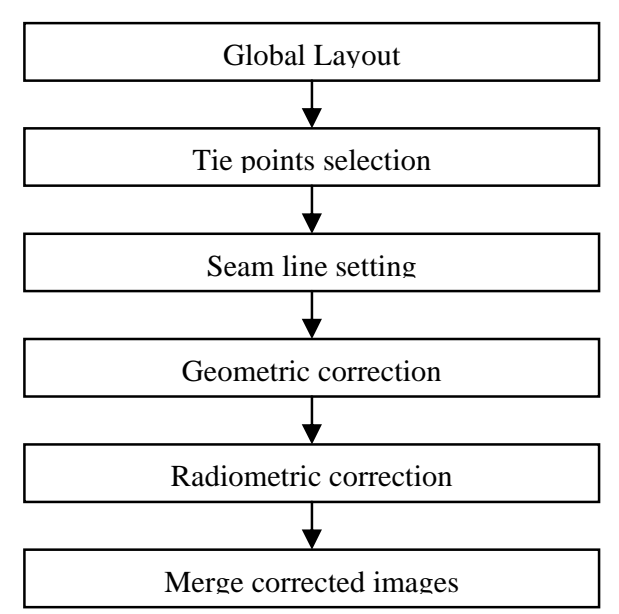

Fig. 1. Mosaic 6 steps

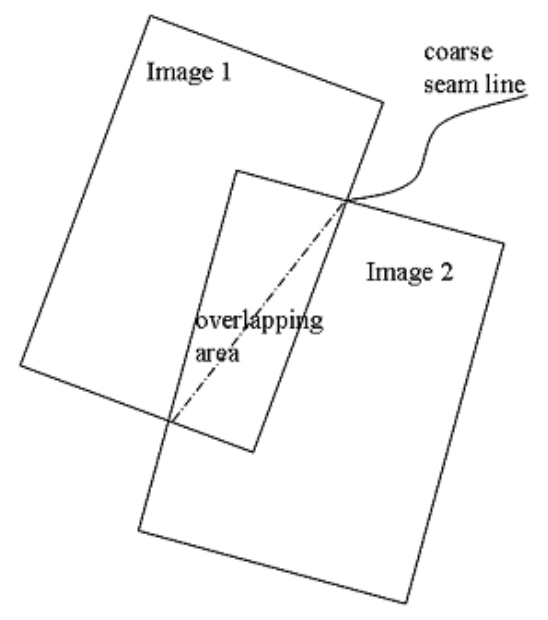

Fig. 2. Global layout phase

\subsection{Tie point selection phase}

Tie point selection phase is to extract the tie point from the overlapping area of two images like figure 3 .

\subsection{Seam line setting phase}

Seam line setting phase is to select precise seam line like figure 4 .

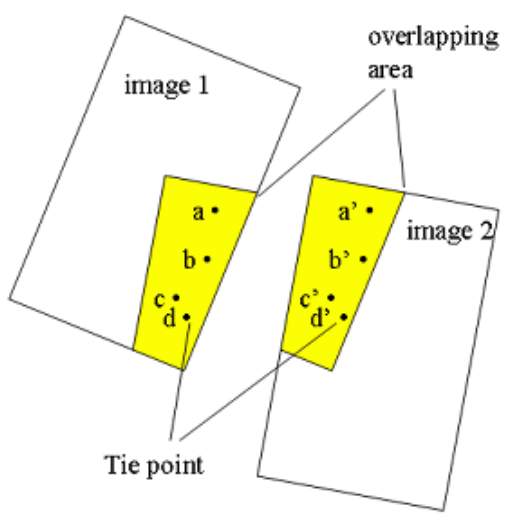

Fig. 3. Tie point selection

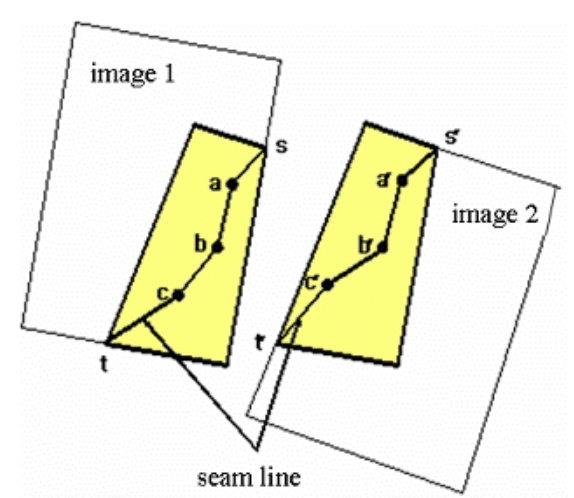

Fig. 4. Seam line setting

Automation theory adds up the result of 4 factors with weight. 4 factors are like next.

1. distance between near two tie points

2. distance between coarse seam line and tie point

3. distance between pair left/right tie points

4. distance between near two pair left/right tie points

\subsection{Geometric correction phase}

Geometric correction phase is to achieve the geometric correction like figure 5 .

Mean seam line of figure 5 (b) is a single line that connects pair tie point by mean coordinate. The seam line area of left and right images are corrected as mean seam line area. 


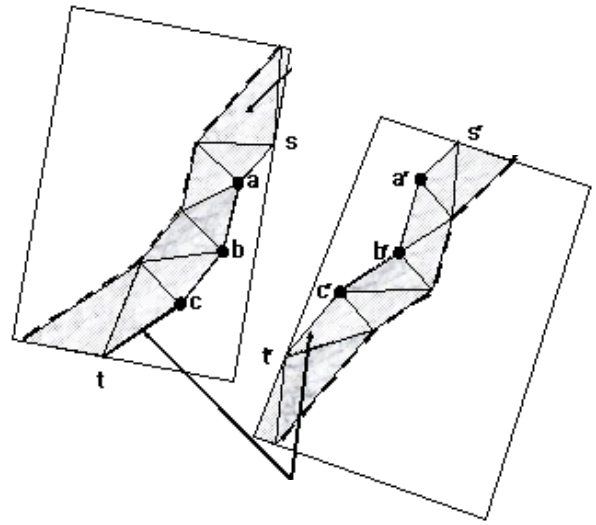

(a) Before the correction

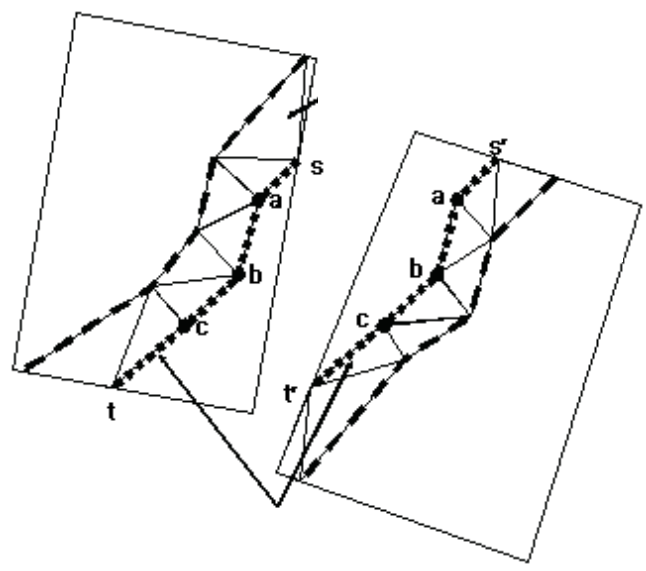

(b) After the correction

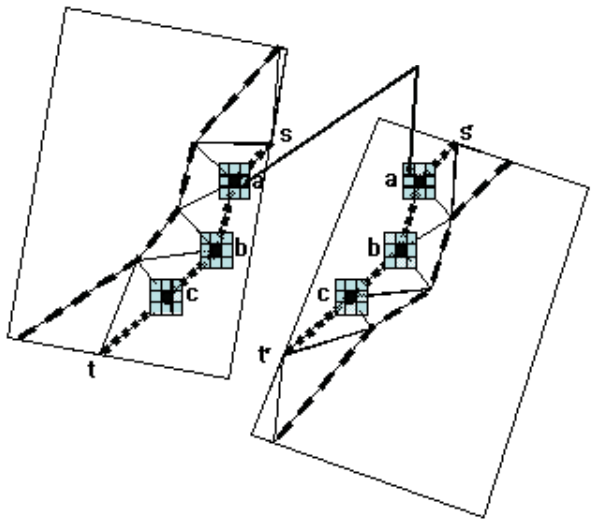

Fig. 6. Radiometric correction

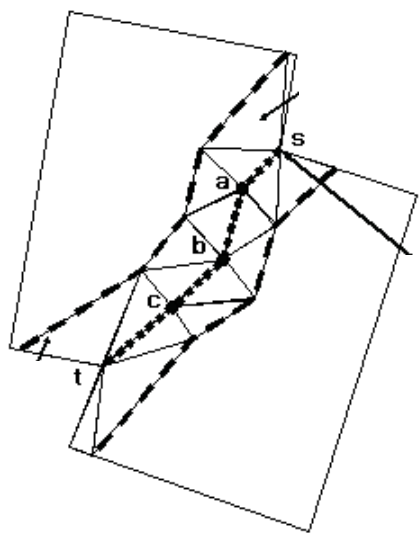

Fig. 7. Merge corrected images

Fig. 5. Geometric correction

\section{Proposed algorithm}

\subsection{Radiometric correction phase}

Radiometric correction phase is to achieve the radiometric correction. Radiometric correction is to remove the radiometric seam happened to mean seam line $[1,10]$ like figure 6.

\subsection{Merge corrected images phase}

Merge corrected images phase is to merge the unchanged area and changed correction area in two inserted images like figure 7 .

\subsection{FOM measurement}

This paper proposes the method that is to consider FOM(Figure Of Merit) as the fifth factor to generate more practical mosaic seam line. FOM shows the identification of a tie point. The core of proposed algorithm is the method how to give the degree of FOM like theorem 1. To give the degree of FOM about the tie point, we consider the correlation and direction of pair tie point like following 6 steps.

$$
F O M=\left(\frac{\text { correlation }+ \text { direction }}{2}\right) \cdot 100
$$

(Theorem 1) 
The first step selects 8 points in the area of original and target images tie point as an image patch like figure 8 . In figure 8 , $\mathrm{O}$ means the original image and $\mathrm{T}$ means the target image.

\begin{tabular}{|c|c|c|}
\hline $\mathrm{O}_{1}$ & $\mathrm{O}_{2}$ & $\mathrm{O}_{3}$ \\
\hline $\mathrm{O}_{4}$ & $\begin{array}{c}\text { Tie point } \\
\mathrm{O}_{5}\end{array}$ & $\mathrm{O}_{6}$ \\
\hline $\mathrm{O}_{7}$ & $\mathrm{O}_{6}$ & $\mathrm{Og}_{9}$ \\
\hline
\end{tabular}

\begin{tabular}{|c|c|c|}
\hline $\mathrm{T}_{1}$ & $\mathrm{~T}_{\mathbf{2}}$ & $\mathrm{T}_{3}$ \\
\hline $\mathrm{T}_{\mathbf{4}}$ & $\begin{array}{c}\text { Tie point } \\
\mathrm{T}_{\mathbf{5}}\end{array}$ & $\mathrm{T}_{\mathbf{6}}$ \\
\hline $\mathrm{T}_{7}$ & $\mathrm{~T}_{\mathbf{8}}$ & $\mathrm{T}_{\mathbf{9}}$ \\
\hline
\end{tabular}

Fig. 8. Image Patch

The second step takes the mean $\mu$ and standard deviation $\sigma$ of both images like theorem 2 and 3

$$
\begin{gathered}
\mu_{O}=\frac{\sum_{i=1}^{9} O_{i}}{9}, \sigma_{O^{2}}=\frac{\sum_{i=1}^{9} O_{i}^{2}}{9}-\left[\frac{\sum_{i=1}^{9} O_{i}}{9}\right]^{2} \text { (Theorem 2) } \\
\mu_{T}=\frac{\sum_{i=1}^{9} T_{i}}{9}, \sigma_{T^{2}}=\frac{\sum_{i=1}^{9} T_{i}^{2}}{9}-\left[\frac{\sum_{i=1}^{9} T_{i}}{9}\right]^{2} \text { (Theorem 3) }
\end{gathered}
$$

The third step is to normalize both images like theorem 4 and 5. Accordingly, the mean and distribution of $3 \times 3$ image patch become respectively 0 and 1 .

$$
\begin{gathered}
\forall_{i}=1 . .9, O_{i}=\frac{O_{i}-\mu_{O}}{\sigma_{O}} \text { (Theorem 4) } \\
\forall_{i}=1 . .9, T_{i}=\frac{T_{i}-\mu_{T}}{\sigma_{T}} \text { (Theorem 5) }
\end{gathered}
$$

The fourth step computes the correlation $\mathrm{FOM}_{1}$ of an image patch like theorem 6 . The value of computed $\mathrm{FOM}_{1}$ is from 0 to 1 .

$$
\mathrm{FOM}_{1}=\frac{\left|\sum_{i=1}^{9} O_{i} \cdot T_{i}\right|}{\sqrt{\sum_{i=1}^{9} O_{i}^{2} \cdot \sum_{i=1}^{9} T_{i}^{2}}}
$$

The fifth step computes the direction $\mathrm{FOM}_{2}$ of an image patch like theorem 7 13. The value of computed $\mathrm{FOM}_{2}$ is also from 0 to 1 . Theorem 7 9 is identically applied to target image.

$$
\begin{array}{r}
X_{O}=\left(O_{3}+2 O_{6}+O_{9}\right)-\left(O_{1}+2 O_{4}+O_{7}\right) \text { (Theorem 7) } \\
Y_{O}=\left(O_{1}+2 O_{2}+O_{3}\right)-\left(O_{7}+2 O_{8}+O_{9}\right) \text { (Theorem 8) } \\
\text { Angle }_{O}=a \tan 2\left(\frac{Y_{O}}{X_{O}}\right) \text { (Theorem 9) } \\
\text { FOM }_{2}=\mid \text { Angle }_{O}-\text { Angle }_{T} \mid \text { (Theorem 10) } \\
\text { IfFOM } 2>\pi, \text { FOM }_{2}=2 \pi-\text { FOM }_{2} \text { (Theorem 11) } \\
\text { FOM }_{2}=\frac{\text { FOM }_{2}}{\pi} \text { (Theorem 12) } \\
\text { FOM }_{2}=1-\text { FOM }_{2} \text { (Theorem 13) }
\end{array}
$$

The sixth step adds a correlation $\mathrm{FOM}_{1}$ to a direction $\mathrm{FOM}_{2}$, get the mean of both and multiply it by 100 like theorem 14.

$$
F O M=\left(\frac{F O M_{1}+F O M_{2}}{2}\right) \cdot 100(\text { Theorem 14) }
$$

Accordingly, four factors of section 2 and FOM are taken into account for weights of the graph in the generation process of seam line.

\subsection{Dijkstra algorithm improvement}

Standard Dijkstra algorithm retrieves the shortest path from directed graph with weight, which is positive constant. However, improved Dijkstra algorithm may be utilized if the weight of each edge is not a fixed constant $[3,11]$.

All 5 factors have the same level of importance. So, all factors must be equally considered without discrimination. However, the values of 5 factors do not have the same range. When we actually measure the first factor (distance between near two tie points), we get the wide range value as $84 \sim 972$ but FOM is limited to $0 \sim 100$. To solve this problem, we achieve the fine adjustment of factors and Dijkstra algorithm repeatedly so that the value of 5 factors could be considered for generating a seam line. That is to say, we retrieve repeatedly the shortest path until 5 factors of artery existed in the selected path get similar value, and approve the retrieved shortest path as a seam line. 


\section{Experiment and consideration}

\subsection{Test data}

Figure 9 shows two images to exercise the proposed algorithm. Two illustrated spatial ortho-images include the overlapping area and vector illustrated in spatial orthoimage is a single relief map. The right image becomes distorted geometrically. Figure 10 shows the tie point to test the proposed algorithm and the number of tie point is 15 without a starting and ending point.

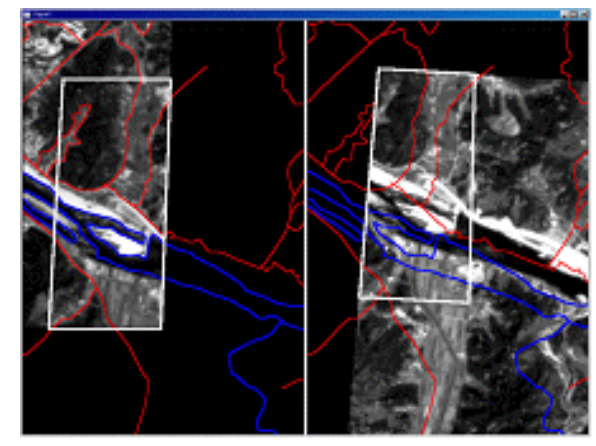

Fig. 9. Test image

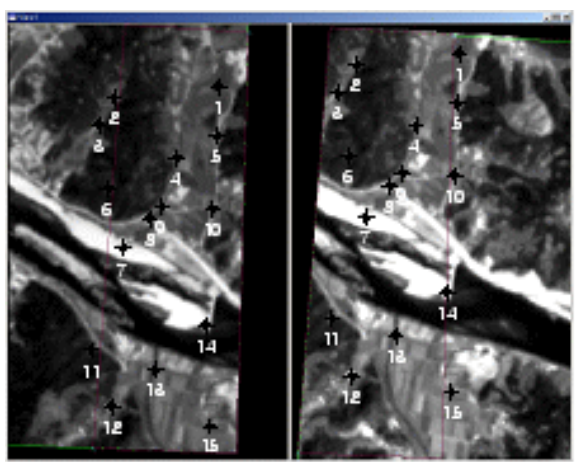

Fig. 10. Tie point

\subsection{Weight assignment}

In previous section, we defined the test data. The test data is showed as a directed graph $\mathrm{G}$ like figure 11 .

Directed graph $G=(V, E)$ is defined like following.

Firstly, tie point becomes a vertex.
Secondly, the line that connects tie point become an artery and has the direction.

Thirdly, the weight assigned to an artery is the sum of 5 factors value.

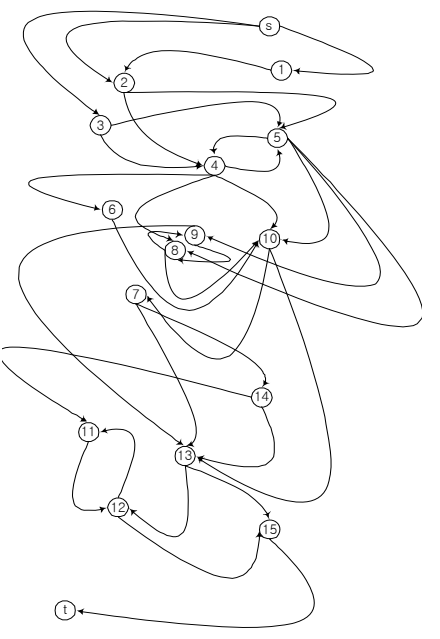

Fig. 11. Directed graph G

Figure 12 shows two random vertexes of directed graph $\mathrm{v}$ and $\mathrm{u}$. Also, the values of 5 factors are measured using following methods.
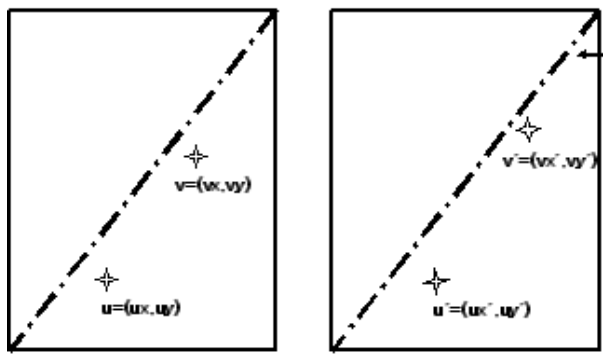

Fig. 12. Two random vertexes

Distance between two near tie points is $F_{1}$ that is measured by theorem 15 , and table 1 shows the result.

$$
F_{1}=\sqrt{(v x-u x)^{2}+(v y-u y)^{2}}(\text { Theorem 15) }
$$

Distance between coarse seam line and tie point is $\mathrm{F}_{2}$ that is measured by theorem 16, and table 2 shows the result.

$$
F_{2}=\sqrt{(u x-p)^{2}+(u y-q)^{2}} \text { (Theorem 16) }
$$


Table 1. $F_{1}$ result

\begin{tabular}{|c|c|c|c|c|c|c|c|c|c|c|c|c|c|c|c|c|c|}
\hline & $\mathrm{s}$ & 1 & 2 & 3 & 4 & 5 & 6 & 7 & 8 & 9 & 10 & 11 & 12 & 13 & 14 & 15 & $\mathrm{t}$ \\
\hline $\mathrm{s}$ & 0 & 327 & 814 & 972 & 0 & 0 & 0 & 0 & 0 & 0 & 0 & 0 & 0 & 0 & 0 & 0 & 0 \\
\hline 1 & 0 & 0 & 590 & 0 & 0 & 0 & 0 & 0 & 0 & 0 & 0 & 0 & 0 & 0 & 0 & 0 & 0 \\
\hline 2 & 0 & 0 & 0 & 0 & 478 & 614 & 0 & 0 & 0 & 0 & 0 & 0 & 0 & 0 & 0 & 0 & 0 \\
\hline 3 & 0 & 0 & 0 & 0 & 480 & 678 & 0 & 0 & 0 & 0 & 0 & 0 & 0 & 0 & 0 & 0 & 0 \\
\hline 4 & 0 & 0 & 0 & 0 & 0 & 259 & 427 & 0 & 360 & 0 & 347 & 0 & 0 & 0 & 0 & 0 & 0 \\
\hline 5 & 0 & 0 & 0 & 0 & 259 & 0 & 0 & 0 & 586 & 502 & 401 & 0 & 0 & 0 & 0 & 0 & 0 \\
\hline 6 & 0 & 0 & 0 & 0 & 0 & 0 & 0 & 0 & 0 & 0 & 607 & 0 & 0 & 0 & 0 & 0 & 0 \\
\hline 7 & 0 & 0 & 0 & 0 & 0 & 0 & 0 & 0 & 0 & 0 & 0 & 0 & 0 & 679 & 634 & 0 & 0 \\
\hline 8 & 0 & 0 & 0 & 0 & 0 & 0 & 0 & 0 & 0 & 84 & 354 & 0 & 0 & 0 & 0 & 0 & 0 \\
\hline 9 & 0 & 0 & 0 & 0 & 0 & 0 & 0 & 0 & 84 & 0 & 0 & 0 & 0 & 890 & 0 & 0 & 0 \\
\hline 10 & 0 & 0 & 0 & 0 & 0 & 0 & 0 & 553 & 0 & 0 & 0 & 0 & 0 & 938 & 0 & 0 & 0 \\
\hline 11 & 0 & 0 & 0 & 0 & 0 & 0 & 0 & 0 & 0 & 0 & 0 & 0 & 331 & 0 & 0 & 0 & 0 \\
\hline 12 & 0 & 0 & 0 & 0 & 0 & 0 & 0 & 0 & 0 & 0 & 0 & 331 & 0 & 0 & 0 & 580 & 0 \\
\hline 13 & 0 & 0 & 0 & 0 & 0 & 0 & 0 & 0 & 0 & 0 & 0 & 0 & 330 & 0 & 0 & 453 & 0 \\
\hline 14 & 0 & 0 & 0 & 0 & 0 & 0 & 0 & 0 & 0 & 0 & 0 & 667 & 0 & 371 & 0 & 0 & 0 \\
\hline 15 & 0 & 0 & 0 & 0 & 0 & 0 & 0 & 0 & 0 & 0 & 0 & 0 & 0 & 0 & 0 & 0 & 536 \\
\hline $\mathrm{t}$ & 0 & 0 & 0 & 0 & 0 & 0 & 0 & 0 & 0 & 0 & 0 & 0 & 0 & 0 & 0 & 0 & 0 \\
\hline
\end{tabular}

Table 2. $F_{2}$ result

\begin{tabular}{|c|c|c|c|c|c|c|c|c|c|c|c|c|c|c|c|c|c|}
\hline & $\mathrm{s}$ & 1 & 2 & 3 & 4 & 5 & 6 & 7 & 8 & 9 & 10 & 11 & 12 & 13 & 14 & 15 & $\mathrm{t}$ \\
\hline $\mathrm{s}$ & 0 & 49 & 588 & 636 & 0 & 0 & 0 & 0 & 0 & 0 & 0 & 0 & 0 & 0 & 0 & 0 & 0 \\
\hline 1 & 0 & 0 & 588 & 0 & 0 & 0 & 0 & 0 & 0 & 0 & 0 & 0 & 0 & 0 & 0 & 0 & 0 \\
\hline 2 & 0 & 0 & 0 & 0 & 157 & 25 & 0 & 0 & 0 & 0 & 0 & 0 & 0 & 0 & 0 & 0 & 0 \\
\hline 3 & 0 & 0 & 0 & 0 & 157 & 25 & 0 & 0 & 0 & 0 & 0 & 0 & 0 & 0 & 0 & 0 & 0 \\
\hline 4 & 0 & 0 & 0 & 0 & 0 & 25 & 478 & 0 & 193 & 0 & 124 & 0 & 0 & 0 & 0 & 0 & 0 \\
\hline 5 & 0 & 0 & 0 & 0 & 157 & 0 & 0 & 0 & 193 & 154 & 124 & 0 & 0 & 0 & 0 & 0 & 0 \\
\hline 6 & 0 & 0 & 0 & 0 & 0 & 0 & 0 & 0 & 0 & 0 & 124 & 0 & 0 & 0 & 0 & 0 & 0 \\
\hline 7 & 0 & 0 & 0 & 0 & 0 & 0 & 0 & 0 & 0 & 0 & 0 & 0 & 0 & 86 & 292 & 0 & 0 \\
\hline 8 & 0 & 0 & 0 & 0 & 0 & 0 & 0 & 0 & 0 & 154 & 124 & 0 & 0 & 0 & 0 & 0 & 0 \\
\hline 9 & 0 & 0 & 0 & 0 & 0 & 0 & 0 & 0 & 193 & 0 & 0 & 0 & 0 & 86 & 0 & 0 & 0 \\
\hline 10 & 0 & 0 & 0 & 0 & 0 & 0 & 0 & 287 & 0 & 0 & 0 & 0 & 0 & 86 & 0 & 0 & 0 \\
\hline 11 & 0 & 0 & 0 & 0 & 0 & 0 & 0 & 0 & 0 & 0 & 0 & 0 & 86 & 0 & 0 & 0 & 0 \\
\hline 12 & 0 & 0 & 0 & 0 & 0 & 0 & 0 & 0 & 0 & 0 & 0 & 288 & 0 & 0 & 0 & 489 & 0 \\
\hline 13 & 0 & 0 & 0 & 0 & 0 & 0 & 0 & 0 & 0 & 0 & 0 & 0 & 86 & 0 & 0 & 489 & 0 \\
\hline 14 & 0 & 0 & 0 & 0 & 0 & 0 & 0 & 0 & 0 & 0 & 0 & 288 & 0 & 86 & 0 & 0 & 0 \\
\hline 15 & 0 & 0 & 0 & 0 & 0 & 0 & 0 & 0 & 0 & 0 & 0 & 0 & 0 & 0 & 0 & 0 & 0 \\
\hline $\mathrm{t}$ & 0 & 0 & 0 & 0 & 0 & 0 & 0 & 0 & 0 & 0 & 0 & 0 & 0 & 0 & 0 & 0 & 0 \\
\hline
\end{tabular}

Table 3. $F_{3}$ result

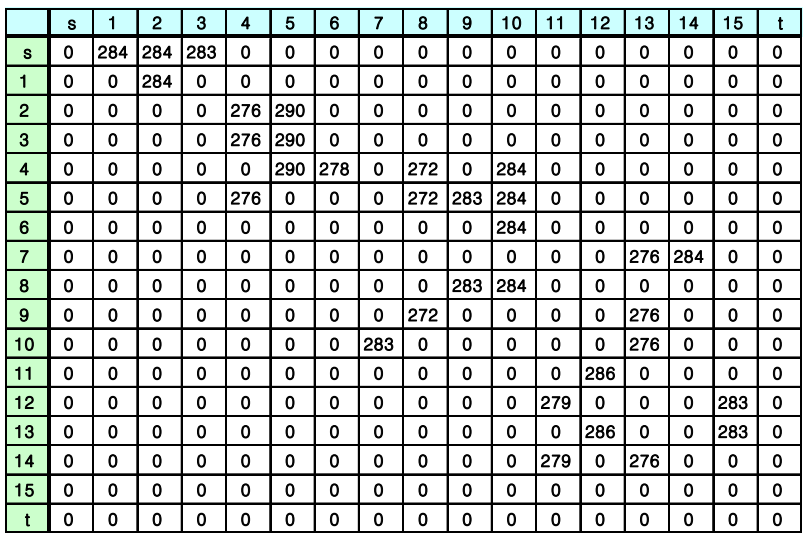

Table $4 . \mathrm{F}_{4}$ result

\begin{tabular}{|c|c|c|c|c|c|c|c|c|c|c|c|c|c|c|c|c|c|}
\hline & $\mathrm{s}$ & 1 & 2 & 3 & 4 & 5 & 6 & 7 & 8 & 9 & 10 & 11 & 12 & 13 & 14 & 15 & $\mathrm{t}$ \\
\hline $\mathrm{s}$ & 0 & 284 & 284 & 283 & 0 & 0 & 0 & 0 & 0 & 0 & 0 & 0 & 0 & 0 & 0 & 0 & 0 \\
\hline 1 & 0 & 0 & 5 & 0 & 0 & 0 & 0 & 0 & 0 & 0 & 0 & 0 & 0 & 0 & 0 & 0 & 0 \\
\hline 2 & 0 & 0 & 0 & 0 & 9 & 8 & 0 & 0 & 0 & 0 & 0 & 0 & 0 & 0 & 0 & 0 & 0 \\
\hline 3 & 0 & 0 & 0 & 0 & 10 & 16 & 0 & 0 & 0 & 0 & 0 & 0 & 0 & 0 & 0 & 0 & 0 \\
\hline 4 & 0 & 0 & 0 & 0 & 0 & 16 & 5 & 0 & 5 & 0 & 8 & 0 & 0 & 0 & 0 & 0 & 0 \\
\hline 5 & 0 & 0 & 0 & 0 & 16 & 0 & 0 & 0 & 21 & 10 & 9 & 0 & 0 & 0 & 0 & 0 & 0 \\
\hline 6 & 0 & 0 & 0 & 0 & 0 & 0 & 0 & 0 & 0 & 0 & 8 & 0 & 0 & 0 & 0 & 0 & 0 \\
\hline 7 & 0 & 0 & 0 & 0 & 0 & 0 & 0 & 0 & 0 & 0 & 0 & 0 & 0 & 7 & 2 & 0 & 0 \\
\hline 8 & 0 & 0 & 0 & 0 & 0 & 0 & 0 & 0 & 0 & 11 & 12 & 0 & 0 & 0 & 0 & 0 & 0 \\
\hline 9 & 0 & 0 & 0 & 0 & 0 & 0 & 0 & 0 & 11 & 0 & 0 & 0 & 0 & 7 & 0 & 0 & 0 \\
\hline 10 & 0 & 0 & 0 & 0 & 0 & 0 & 0 & 1 & 0 & 0 & 0 & 0 & 0 & 8 & 0 & 0 & 0 \\
\hline 11 & 0 & 0 & 0 & 0 & 0 & 0 & 0 & 0 & 0 & 0 & 0 & 0 & 8 & 0 & 0 & 0 & 0 \\
\hline 12 & 0 & 0 & 0 & 0 & 0 & 0 & 0 & 0 & 0 & 0 & 0 & 8 & 0 & 0 & 0 & 11 & 0 \\
\hline 13 & 0 & 0 & 0 & 0 & 0 & 0 & 0 & 0 & 0 & 0 & 0 & 0 & 11 & 0 & 0 & 10 & 0 \\
\hline 14 & 0 & 0 & 0 & 0 & 0 & 0 & 0 & 0 & 0 & 0 & 0 & 5 & 0 & 9 & 0 & 0 & 0 \\
\hline 15 & 0 & 0 & 0 & 0 & 0 & 0 & 0 & 0 & 0 & 0 & 0 & 0 & 0 & 0 & 0 & 0 & 283 \\
\hline $\mathrm{t}$ & 0 & 0 & 0 & 0 & 0 & 0 & 0 & 0 & 0 & 0 & 0 & 0 & 0 & 0 & 0 & 0 & 0 \\
\hline
\end{tabular}

Table 5. $\mathrm{F}_{5}$ result

\begin{tabular}{|c|c|c|c|c|c|c|c|c|c|c|c|c|c|c|c|c|c|}
\hline & $\mathrm{s}$ & 1 & 2 & 3 & 4 & 5 & 6 & 7 & 8 & 9 & 10 & 11 & 12 & 13 & 14 & 15 & $\mathrm{t}$ \\
\hline $\mathrm{s}$ & 0 & 30 & 40 & 45 & 0 & 0 & 0 & 0 & 0 & 0 & 0 & 0 & 0 & 0 & 0 & 0 & 0 \\
\hline 1 & 0 & 0 & 40 & 0 & 0 & 0 & 0 & 0 & 0 & 0 & 0 & 0 & 0 & 0 & 0 & 0 & 0 \\
\hline 2 & 0 & 0 & 0 & 0 & 40 & 10 & 0 & 0 & 0 & 0 & 0 & 0 & 0 & 0 & 0 & 0 & 0 \\
\hline 3 & 0 & 0 & 0 & 0 & 40 & 10 & 0 & 0 & 0 & 0 & 0 & 0 & 0 & 0 & 0 & 0 & 0 \\
\hline 4 & 0 & 0 & 0 & 0 & 0 & 10 & 45 & 0 & 5 & 0 & 90 & 0 & 0 & 0 & 0 & 0 & 0 \\
\hline 5 & 0 & 0 & 0 & 0 & 40 & 0 & 0 & 0 & 5 & 5 & 90 & 0 & 0 & 0 & 0 & 0 & 0 \\
\hline 6 & 0 & 0 & 0 & 0 & 0 & 0 & 0 & 0 & 0 & 0 & 90 & 0 & 0 & 0 & 0 & 0 & 0 \\
\hline 7 & 0 & 0 & 0 & 0 & 0 & 0 & 0 & 0 & 0 & 0 & 0 & 0 & 0 & 30 & 20 & 0 & 0 \\
\hline 8 & 0 & 0 & 0 & 0 & 0 & 0 & 0 & 0 & 0 & 5 & 90 & 0 & 0 & 0 & 0 & 0 & 0 \\
\hline 9 & 0 & 0 & 0 & 0 & 0 & 0 & 0 & 0 & 5 & 0 & 0 & 0 & 0 & 30 & 0 & 0 & 0 \\
\hline 10 & 0 & 0 & 0 & 0 & 0 & 0 & 0 & 5 & 0 & 0 & 0 & 0 & 0 & 30 & 0 & 0 & 0 \\
\hline 11 & 0 & 0 & 0 & 0 & 0 & 0 & 0 & 0 & 0 & 0 & 0 & 0 & 45 & 0 & 0 & 0 & 0 \\
\hline 12 & 0 & 0 & 0 & 0 & 0 & 0 & 0 & 0 & 0 & 0 & 0 & 30 & 0 & 0 & 0 & 20 & 0 \\
\hline 13 & 0 & 0 & 0 & 0 & 0 & 0 & 0 & 0 & 0 & 0 & 0 & 0 & 45 & 0 & 0 & 20 & 0 \\
\hline 14 & 0 & 0 & 0 & 0 & 0 & 0 & 0 & 0 & 0 & 0 & 0 & 30 & 0 & 30 & 0 & 0 & 0 \\
\hline 15 & 0 & 0 & 0 & 0 & 0 & 0 & 0 & 0 & 0 & 0 & 0 & 0 & 0 & 0 & 0 & 0 & 0 \\
\hline $\mathrm{t}$ & 0 & 0 & 0 & 0 & 0 & 0 & 0 & 0 & 0 & 0 & 0 & 0 & 0 & 0 & 0 & 0 & 0 \\
\hline
\end{tabular}

Table 6. The weight of $\mathrm{G}$

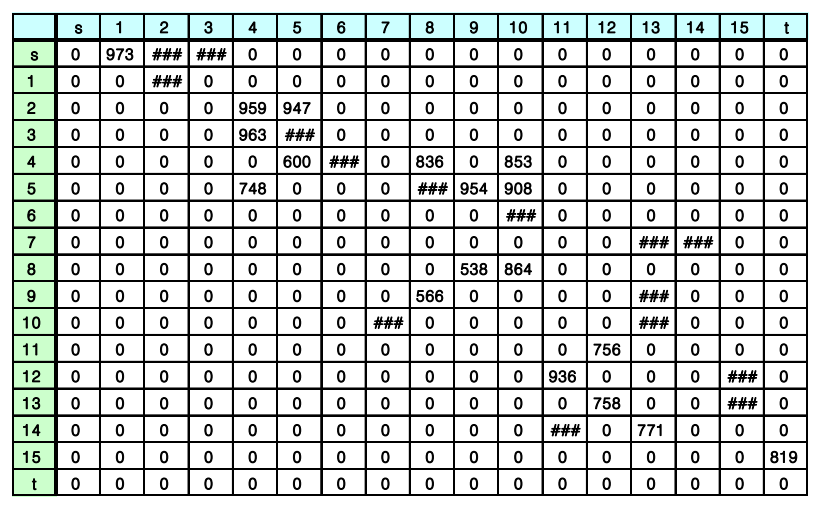


Distance between left and right tie points is $F_{3}$ that is measured by theorem 17 , and table 3 shows the result.

$$
F_{3}=\sqrt{\left(u x-u x^{\prime}\right)^{2}+\left(u y-u y^{\prime}\right)^{2}} \text { (Theorem 17) }
$$

Distance between two near tie points pair is $F_{4}$ that is measured by theorem 18 , and table 4 shows the result.

$$
F_{4}=\sqrt{\left(\left|v x-v x^{\prime}\right|+\left|u x-u x^{\prime}\right|\right)^{2}+\left(\left|v y-v y^{\prime}\right|+\left|u y-u y^{\prime}\right|\right)^{2}}
$$

(Theorem 18)

FOM $F_{5}$ is measured by theorem 1 and table 5 shows the result.

The weight of directed graph $\mathrm{G}$ is $w$ that is measured by theorem 19, and table 6 shows the result.

$$
w=F_{1}+F_{2}+F_{3}+F_{4}+F_{5} \text { (Theorem 19) }
$$

\subsection{Seam line generation}

The conditions of 5 factors are like followings.

Firstly, distance between two near tie points must be short. Actually, the overlapping area of mosaic image that use tie points scattered over wide area as a seam line may has the cutting of relief.

Secondly, distance between coarse seam line and tie point must be short. To select the tie point near to a coarse seam line is to achieve considerations at the setting of coarse seam line.

Thirdly, distance between left and right tie points must be short. This means that we must select the tie point existed in the area of small geometrical distortion for minimizing the geometrical correction range. If the geometrical distortion between left and right tie points is large, very large area need to achieve geometrical correction and may change the area not to need correction.

Fourthly, distance between two near left and right tie points must be short. This means that we must select the point that is lower distortion between two near left and right tie points for achieving geometrical correction by same direction and similar size. Minimum geometrical movement has influence on running speed and quality of mosaic image.

Fifthly, the level of FOM must be higher. High FOM means that the identification of tie point is easier and has high probability to be selected when human workers generate a seam line by hand. If we generate a seam line after selecting the tie point of high FOM, we can make similar result compared to a seam line generated by human workers.

This paper implements above 5 factors in the directed graph $G$ as a test data. We achieve repeatedly test by 3 times. These repeated processes are in table 7 and figure $13 \sim 15$.

Table 7. Repeated test process

\begin{tabular}{|c|r|r|r|}
\hline & 1 time repetition & 2 times repetition & 3 times repetiton \\
\hline \hline coef1 & 1.0 & 2.0 & 8.5 \\
\hline coef2 & 1.0 & 5.0 & 24.2 \\
\hline coef3 & 1.0 & 5.2 & 22.9 \\
\hline coef4 & 1.0 & 12.0 & 53.9 \\
\hline coef5 & 1.0 & 32.9 & 309.1 \\
\hline sum1 & 3566.6 & 7725.5 & 32451.2 \\
\hline sum2 & 1443.9 & 6724.5 & 32451.2 \\
\hline sum3 & 1402.3 & 7304.0 & 32451.2 \\
\hline sum4 & 601.4 & 7244.5 & 32451.2 \\
\hline sum5 & 220.0 & 3452.8 & 32451.2 \\
\hline total & 7234.2 & 32451.3 & 162256.0 \\
\hline \hline $\begin{array}{c}\text { selection } \\
\text { path }\end{array}$ & $\mathrm{s} 24101315 \mathrm{t}$ & $\mathrm{s} 2591315 \mathrm{t}$ & $\mathrm{s} 2591315 \mathrm{t}$ \\
\hline
\end{tabular}

Figure 13 shows the result of 1 time repetition by proposed algorithm. Table 7 shows the result of program running and figure 13 shows visually the generated seam line. We try to run the 2nd test because the measured values of 5 factors are not reflected.

Figure 14 shows the result of 2 times repetition and visually the generated seam line. We try to run the 3rd test because the measured values of 5 factors are not reflected. Figure 15 shows the result of 3 times repetition and visually the generated seam line. Seam line selected in the 3rd test is identical to the seam line selected in the 2nd test. This means that the seam line is identical although we give the weight by the corrected coefficient based on the result of 2 times test.

The measured values of 5 factors may be reflected, so we stop a continual running test and take the shortest path $\mathrm{P}$ of 3rd test as a seam line of directed graph G.

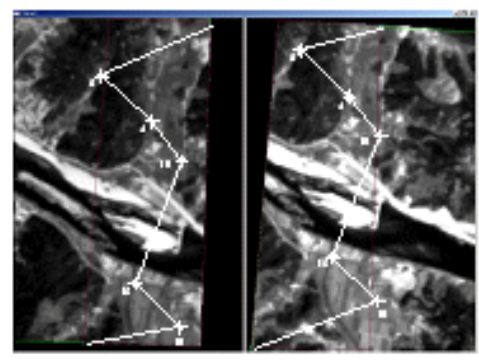

Fig. 13. The seam line of first test 


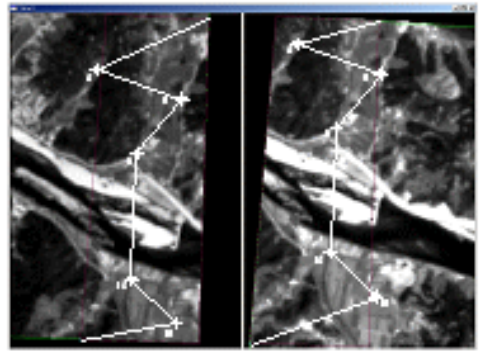

Fig. 14. The seam line of second test

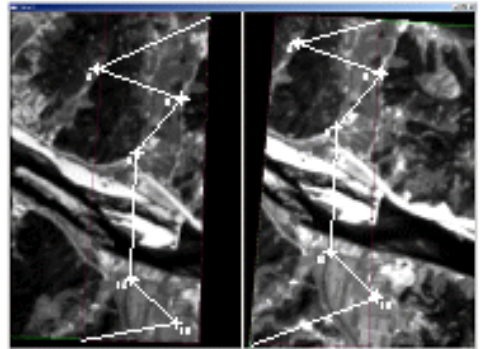

Fig. 15. The seam line of third test

\subsection{Availability evaluation}

We define the test data, give the weight, and generate a seam line through the experiment so far. The availability evaluation of generated seam line is compared with another case seam line. We compare the evaluation items of 3 cases such as continual running test of 5 factors, 4 factors running test by automation theory, and simple distance that considers only distance with weight.

The evaluation items are the generation time of seam line, running time of geometrical correction, and mosaic result. Following is the evaluation result.

(1)Generation time of seam line

Figure 16 shows the result of 3 cases.

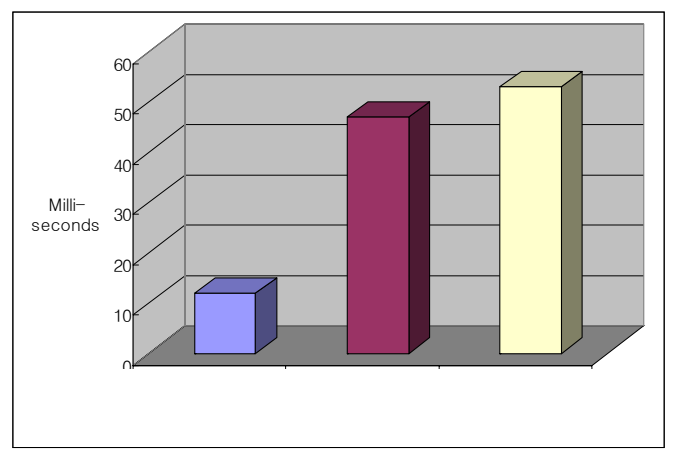

Fig. 16. Generation time of seam line
In the respect of generation time of seam line, "simple distance" case that consider only distance with weight is 12 milliseconds, " 4 factors running test" is 47 milliseconds, " 5 factors running test by this paper" is 53 milliseconds. The difference between 4 factors and 5 factors method is very little.

In actual same test data, "simple distance" generates a seam line by only one running, "4 factors running test" by 2 times running, and " 5 factors running test" by 3 times running. Also, the path of generated seam line is different. Figure 17 shows the difference of 3 cases seam line due to the different tie point selected by 3 cases
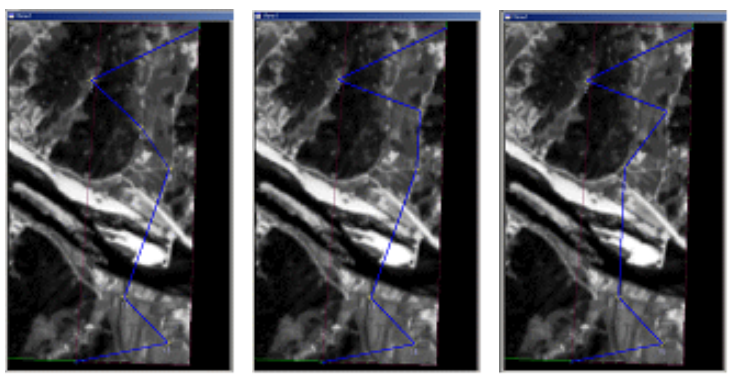

(a) Simple distance (b) 4 factors running (c) 5 factors running

Fig. 17. The seam line of 3 cases

(2) Running time of geometrical correction

We achieve the geometrical correction using different path of seam line and compare the running time. Figure 18 shows the result of geometrical correction.

In the respect of geometrical correction, the running time of " 4 factors running method" is 55 seconds, " 5 factors running method" by this paper is 56 seconds, and "simple distance" is 66 seconds. The difference between 4 factors and 5 factors method is very little.

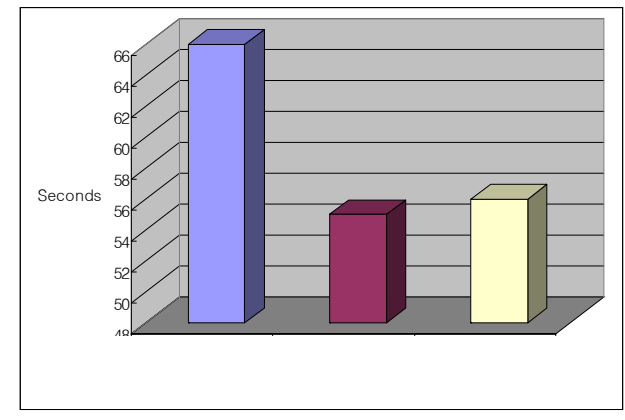

Fig. 18. Running time of geometrical correction 
"4 factors running method" and "5 factors running method" that consider condition to minimize the range of geometrical correction and geometrical movement shows shorter geometrical correction time, but "simple distance" method that only consider the distance without any limitations shows longer geometrical correction time. This is the reason why the seam lines of 3 cases are different one another.

(3) Mosaic result

We achieve the mosaic using different seam line generated by 3 cases and compare the result image of mosaic by user satisfaction

For user satisfaction measurement, we select 20 peoples that have experience about mosaic working and allow them to see the result image of mosaic by the 3 cases of tests. Then 20 peoples may select the good result of case and we compute the selected number. Figure 19 shows the result of user satisfaction measurement.

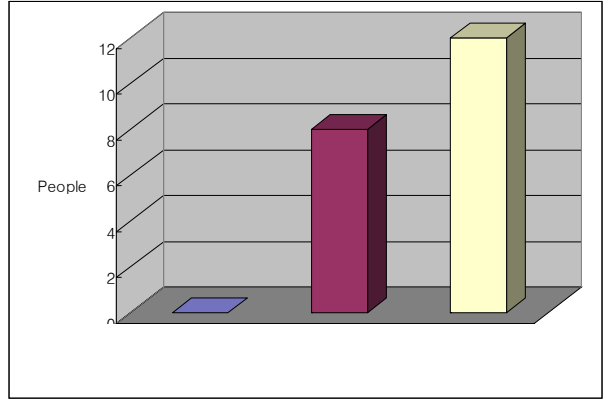

Fig. 19. User satisfaction

Figure 19 shows that all users do not select the mosaic image of "simple distance", 8 users do " 4 factors running method", and 12 users do " 5 factors running method". We know that difference between " 4 factors running method" and " 5 factors running method" is very little by the generation time of seam line and the running time of geometrical correction, but the result of user satisfaction measurement shows that the mosaic image of " 5 factors running method" is better than other methods.

Figure 20 shows the different mosaic images due to the different seam line of 3 cases like figure 17.
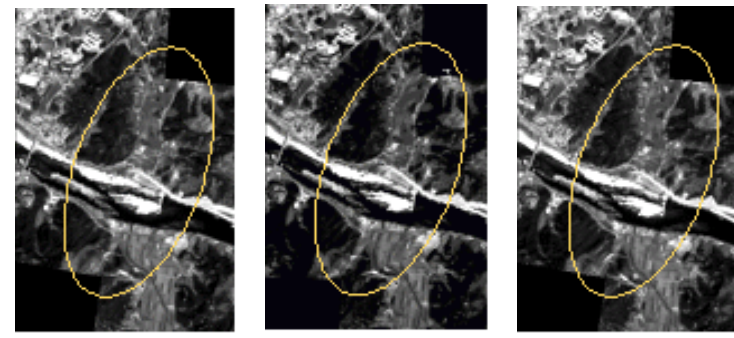

(a) Simple distance (b) 4 factors running (c) 5 factors running

Fig. 20. Mosaic image of 3 cases

Figure 21 shows the mosaic image by various test images using " 5 factors running methods"

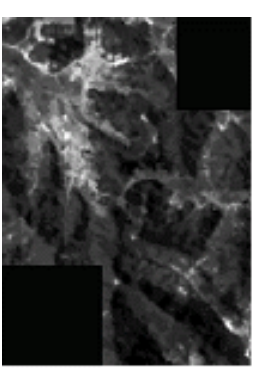

(a) mountainous area

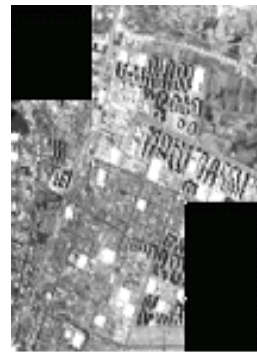

(b) downtown

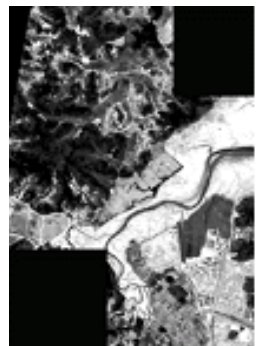

(c) watercourse
Fig. 21. Mosaic image of 5 factors running method

\section{Conclusion}

This paper is to consider the identification of tie point as the fifth factor to generate more practical seam line. That is to say, this paper proposes the generation method of seam line to use 5 factors that includes 4 factors of automation theory and FOM to show the identification of tie point.

For the availability evaluation of proposed algorithm, we compare the items such as the generation time of seam line, running time of geometrical correction, and mosaic image in 3 cases as " 5 factors running method" of this paper, " 4 factors running method" of automation theory, and "simple distance" that consider only distance with weight. We get the next results from these evaluation items.

Firstly, we verify that the mosaic image of "simple distance" do not keep the continuity of feature. 
Secondly, we verify that the seam line, which is generated by the consideration of FOM as the fifth factor, is more practical than the seam line from " 4 factors running method" and "simple distance". In other words, the generation time of seam line and running time of geometrical correction by " 4 factors running method" and "5 factors running method" do not show many difference and the user satisfaction of " 5 factors running method" by mosaic image is better than other cases

Thirdly, FOM is the factor that must be considered at the generation of seam line and efficient method in a nowadays that higher spatial resolution image get popular.

\section{References}

[1]Yong Du, Josef Cihlar, Jean Beaubien, and Rasim Latifovic, "Radiometric Normalization, Compositing, and Quality Control for Satellite High Resolution Image Mosaics over Large Areas", IEEE TRANSACTIONS ON GEOSCIENCE AND REMOTE SENSING, Vol.39, No.3, pp.623-634, 2001

[2]B. Guindon, "Utilization of Landsat pathfinder data creation of large area mosaics", ACSM/ASPRS Annual Convention \& Exposition Technical Papers, Vol.2, pp.144-153, 1995

[3] Yehuda Afek, Ariel Brand, “ Mosaicking of Orthorectified aerial Images" , PE\&RS, Vol.64, No.2, pp.115-125, 1998

[4]Dmitry V. Fedorov, Leila M. Fonseca, Charles Kenney, Bangalore S. Manjunath, "Automatic registration and mosaicking system for remotely sensed imagery", Proc. SPIE, Vol.4885, No.8, pp.444-451, 2003

[5]Martin KERSCHNER, “ Twin Snakes for Determining Seam Lines in Orthoimage Mosaicking” , IAPRS, Vol.33, Part B4, pp.454-461, 2000

[6]J. Cihlar, D. Manak, M. D'Iorio, "Evaluation of compositing algorithms for AVHRR data over land", IEEE TRANSACTIONS ON GEOSCIENCE AND REMOTE SENSING, Vol.32, No.2, pp.427437, 1994

[7]M.-L. Duplaquet, “ Building large image mosaics with invisible seam-lines” , Proc. SPIE, Vol.3387, No.7, pp.369-377, 1998

[8]Fonseca, L.M.G, Manjunath, B.S., "Registration techniques for multisensor remotely sensed imagery", PE\&RS, Vol.62, No.9, pp.1049-1056, 1996

[9]D Milgram, "Computer Methods for Creating Photomosaics" IEEE Transactions in Computers, Vol. C-24, pp.1113-1119, 1975.

[10]Xiaojun Yang, C.P.Lo, "Relative Radiometric Normalization Performance for Change Detection from Multi-Date Satellite Images", PE\&RS, Vol.66, No.8, pp.967-980, 2000

[11] Lim. J. S, "Search the intelligent shortest path, lowest bending path and combinational shortest path," Korea Information Processing Society, Vol. 3, No. 1, pp.43-54, 1996

\section{Biography}

Young-Ku Kang

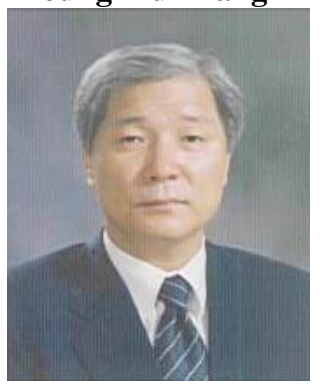

received the B.S. degree in Astronomy Department from Seoul National Univ., Korea, in 1971, and the M.S. degrees in Computer engineering from Florida Institute of Technology, U.S.A, in 1980. He has obtained $\mathrm{PhD}$ in Computer engineering from Soong-sil University, Korea, in 2001. He was a visiting professor at Otago University in New Zealand, 2005-2006. He is a full professor at the department of Computer Engineering, Seoul National University of Technology since 1985 and also his research interests include in the image processing. 